\title{
Crucial involvement of actin filaments in celecoxib and morphine analgesia in a model of inflammatory pain
}

This article was published in the following Dove Press journal:

Journal of Pain Research

8 November 2012

Number of times this article has been viewed

\author{
Patrícia Paiva-Lima' \\ Rafael M Rezende' \\ Rômulo Leite ${ }^{2}$ \\ Igor DG Duarte' \\ YS Bakhle ${ }^{3}$ \\ Janetti N Francischi' \\ 'Department of Pharmacology, \\ Laboratory of Inflammation and \\ Pain, Biological Sciences Institute, \\ Federal University of Minas Gerais, \\ Brazil; ${ }^{2}$ Department of Pharmacology, \\ Faculty of Pharmacy, Federal \\ University of Ouro Preto, Minas \\ Gerais, Brazil; ${ }^{3}$ Leukocyte Biology, \\ National Heart and Lung Institute, \\ Faculty of Medicine, Imperial College, \\ London, UK
}

Background: Celecoxib exerted analgesic effects (hypoalgesia) reversed by opioid receptor antagonists in a model of inflammatory pain. To analyze this celecoxib-induced hypoalgesia further, we assessed the effects of several disruptors of cytoskeletal components in our model of inflammation.

Methods: Hyperalgesia to mechanical stimuli was induced in rat hind paws by intraplantar injection of carrageenan and measured using the Randall-Selitto method over the next 8 hours. The effects of systemic pretreatment with celecoxib and a range of cytoskeletal disruptors (colchicine, nocodazole, cytochalasin B, latrunculin B, acrylamide, each given by intraplantar injection) on carrageenan-induced hyperalgesia were similarly investigated. Morphine and other selective cyclo-oxygenase 1 (SC-560), cyclo-oxygenase 2 (SC-236), and nonselective cyclooxygenase (indomethacin) inhibitors were also tested under similar conditions.

Results: None of the cytoskeletal disruptors affected the peak intensity of carrageenan-induced hyperalgesia, and its duration was increased only by nocodazole and colchicine. Pretreatment with celecoxib 30 minutes before carrageenan reversed the hyperalgesia and raised the nociceptive threshold (hypoalgesia). All analgesic effects of celecoxib were blocked by nocodazole, colchicine, cytochalasin B, and latrunculin B. Pretreatment with morphine also induced hypoalgesia in carrageenan-inflamed paws, an effect reversed by colchicine and cytochalasin $\mathrm{B}$. However, the analgesic effects of indomethacin were not reversed by disruption of actin filaments with cytochalasin B or latrunculin B.

Conclusion: These data strengthen the correlation between cytoskeletal structures and the processes of pain and analgesia.

Keywords: hypoalgesia, celecoxib, morphine, cytoskeleton, actin, cyclo-oxygenases

\section{Introduction}

Celecoxib is classified as a nonsteroidal anti-inflammatory drug (NSAID), and its analgesic and anti-inflammatory actions have been attributed to its inhibition of prostaglandin biosynthesis via cyclo-oxygenase (COX), in common with other NSAIDs, such as indomethacin. ${ }^{1,2}$ We have unexpectedly found that analgesia induced by celecoxib in a model of inflammatory pain was, in contrast with that induced by indomethacin, reversed by opioid receptor antagonists and was not observed in animals tolerant to morphine. ${ }^{3-5}$ We have inferred from these results that the analgesia induced by celecoxib is mediated by endogenous opioids, presumably indirectly, involving release from opioidergic nerves because celecoxib is not a direct agonist of opioid receptors. ${ }^{6}$ However, the mechanistic details of such an indirect action are not known, and in our continuing analysis of celecoxib-induced analgesia, we have investigated the effects
Correspondence: Janetti N Francisch Departamento de Farmacologia, ICB-UFMG, Av Antônio Carlos 6627, Pampulha, Belo Horizonte, MG Brasil, CEP 31901-270

Tel +553134092715

Fax +553134092695

Email janettif@icb.ufmg.br 
of celecoxib on cellular mechanisms known to be involved in antinociception which are not primarily linked to prostaglandin biosynthesis or COX inhibition.

One such mechanism is the rearrangement of intracellular structures, collectively called the cytoskeleton, which appears to be involved in the cellular signaling associated with inflammatory pain. ${ }^{7-9}$ Therefore, we assessed the effects of disruptors of cytoskeletal components and their functions (referred to as cytoskeletal disruptors for brevity) on the analgesia induced by celecoxib in our model of inflammatory pain in rat paws. We studied three types of cytoskeletal disruptors targeted to particular components of the cytoskeleton, ie, nocodazole, and colchicine for microtubules, ${ }^{10,11}$ cytochalasin B and latrunculin B for actin filaments, ${ }^{12-14}$ and acrylamide for intermediate filaments, ${ }^{15,16}$ each given locally into the paw. In this model of hyperalgesia following injection of carrageenan in rat paws, ${ }^{17,18}$ we also used, for comparison, other known analgesic compounds, ie, morphine and NSAIDs, including indomethacin and the SC-560 ${ }^{19}$ and SC-236 ${ }^{2}$ compounds (selective inhibitors of COX-1 and COX-2, respectively).

Our results showed that some, but not all, of the cytoskeletal disruptors interacted with our model, affecting the hyperalgesia induced by carrageenan and the analgesia induced by celecoxib and morphine but not that induced by indomethacin or SC-560. Overall, our results add to the growing evidence for a causal and critical involvement of the cytoskeleton in the processes of nociception and analgesia in the periphery. Furthermore, the similarity of responses to the cytoskeletal disruptors shown by celecoxib and morphine give further support to our proposal that, in this model, the analgesic activity of celecoxib, unlike that of indomethacin, is mediated by endogenous opioids. Some preliminary data from this study have already appeared in abstract form. ${ }^{20}$

\section{Materials and methods}

\section{Animals}

All animal procedures conformed to the guidelines of the International Association for the Study of Pain ${ }^{21}$ and were approved by the Federal University of Minas Gerais ethics committee for animal experimentation (protocol 180/07). Male Holtzman rats weighing 160-190 g were randomly divided into groups of five animals and housed under conditions of a 12-hour light/dark cycle and a temperature of $24^{\circ} \mathrm{C} \pm 2^{\circ} \mathrm{C}$. The animals were allowed free access to food and water. Three days before the experiments, the rats were exposed to the experimental room and apparatus and to handling by the investigator.

\section{Inflammatory pain model}

A standard hyperalgesic effect was produced by intraplantar injection of $\lambda$-carrageenan ${ }^{3} 250 \mu \mathrm{g}$ in $0.1 \mathrm{~mL}$ of sterile saline into one hind paw at time zero. The contralateral paws were injected with the same interplantar volume of saline.

\section{Measurement of mechanical nociceptive response}

Assays to determine the nociceptive thresholds have been described in detail elsewhere., ${ }^{3,422}$ Essentially, we measured the force (in g) applied to the plantar paw surface necessary to trigger hind paw withdrawal using a Basile algesimeter (Ugo Basile SRL, Comerio VA, Italy). ${ }^{23}$ In order to avoid tissue damage, the maximum force applied was limited to $300 \mathrm{~g}$. The hyperalgesic response to carrageenan is shown as a fall in nociceptive thresholds and the term "hypoalgesia" is used to describe the condition when the nociceptive threshold was raised above the basal level, ie, the level at time zero before any treatment of the animal. "Antihyperalgesia" in treated animals is defined as any reversal of hyperalgesia, up to the basal nociceptive threshold. The nociceptive response was measured before, then 30 minutes and one, 2, 3, 4, 6 and 8 hours after carrageenan or saline injections. Results for each paw are presented separately when the nociceptive threshold was affected in both paws, as after morphine (see below). For all experiments showing unilateral effects, the difference $(\Delta)$ between nociceptive thresholds in the right and left paw is presented. Data are presented as time courses.

\section{Hind paw edema measurements}

The volume (in $\mathrm{mL}$ ) of the hind paws for both the vehicle and treated animals was measured with a hydroplethysmometer (Ugo Basile 1750, Italy) at the same time points used for nociceptive threshold measurements, ie, time zero and one, 2, 3, 4, and 6 hours after stimulus injection. Results are presented as the difference $(\Delta$, mean \pm standard error of the mean) in volume between the test (right) paw and the control (left) paw for each group of animals, at the time shown.

\section{Drug doses, route of administration, and schedules \\ Cytoskeletal disruptors}

All compounds were injected in a $0.1 \mathrm{~mL}$ volume of saline via the intraplantar route. We used colchicine $(0.8 \mu \mathrm{g}$ and 8 $\mu \mathrm{g}$ [2.03 nmoles and $20.3 \mathrm{nmoles}])$ and nocodazole $(1 \mu \mathrm{g}$ and $10 \mu \mathrm{g}$ [3.32 nmoles and $33.2 \mathrm{nmoles}]$ ) as disruptors of 
microtubules; latrunculin B $(0.5-5 \mu \mathrm{g}[0.012-1.26$ nmoles $])$ and cytochalasin B (1 ng-1 $\mu \mathrm{g}$ [0.002-2.09 nmoles]) as disruptors of actin filaments and phalloidin $(0.1-10 \mu \mathrm{g}$ [0.13-12.7 nmoles]) as a stabilizer of actin filaments; and acrylamide $(0.1-10 \mu \mathrm{g}$ [1.4-141 nmoles]) as a disruptor of intermediate filaments. All these compounds were stored as stock solutions in dimethyl sulfoxide at $-15^{\circ} \mathrm{C}$. On the day of the experiment, they were diluted with sterile saline; the final maximum concentration of dimethyl sulfoxide was $1 \%$, and this concentration did not affect the nociceptive thresholds.

\section{COX inhibitors}

All compounds were systemically injected s.c. in a volume of $0.1 \mathrm{~mL}$ per $100 \mathrm{~g}$ animal. We used the standard nonselective inhibitor, indomethacin $(4 \mathrm{mg} / \mathrm{kg})$, a selective COX-1 inhibitor, SC-560 $(5 \mathrm{mg} / \mathrm{kg})$, and two selective COX-2 inhibitors, celecoxib $(12 \mathrm{mg} / \mathrm{kg})$ and SC-236 $(12 \mathrm{mg} / \mathrm{kg})$. Indomethacin, SC-560, and SC-236 were dissolved in a mixture of $2 \%$ ethanol, $8 \%$ Tween 80 , and $90 \%$ saline, and celecoxib was administered in saline. ${ }^{3}$

\section{Opioid drugs}

Morphine $2 \mathrm{mg} / \mathrm{kg}$ was dissolved in saline and injected systemically at a volume of $0.1 \mathrm{~mL} / 100 \mathrm{~g}$ animal.

\section{Drug schedules}

Cytoskeletal disruptors were injected into the right hind paw 30 minutes before systemic treatment with COX inhibitors or the opioid agonist, and the same right hind paw received intraplantar carrageenan 30 minutes thereafter. Contralateral paws were injected with the same volume of the corresponding vehicle.

\section{Drug sources}

The selective COX 1 and 2 inhibitors, SC-560 and SC-236, were purchased from Cayman Chemical Company (Ann Arbor, MI), celecoxib (Celebra ${ }^{\mathrm{TM}}$ ) from Pfizer Pharmaceuticals (Caguas, PR), and morphine hydrochloride from Merck AG, (Darmstadt, Germany). All cytoskeletal disruptors were purchased from Sigma-Aldrich (St Louis, MO).

\section{Statistical analysis}

Data are presented as the mean \pm standard error of the mean and analyzed by one-way analysis of variance. Statistical comparisons of means were further examined by Bonferroni's test. Statistical significance was accepted when the difference between means was less than $0.05(P<0.05)$.

\section{Results}

\section{Hyperalgesia and edema induced by intraplantar carrageenan injection}

A standard dose of carrageenan $(250 \mu \mathrm{g}$ per paw) was used in all the experiments, based on our earlier results. ${ }^{3,4}$ This dose induced a characteristic fall in the nociceptive threshold, compared with the contralateral, saline-injected paws, with maximal hyperalgesia reached 2-3 hours after carrageenan injection, returning to normal basal values between 6 and 8 hours (Figure 1). Carrageenan also induced edema, assayed as increased paw volume (Table 1), over the same time course. This aspect of the inflammatory response peaked at 3 hours after carrageenan injection and was still detectable at 6 hours. Paw volumes of the left noninflamed paw which received only saline did not change over the time course of the experiments (data not shown).

\section{Local injection of cytoskeleton disruptors and inflammatory response to carrageenan}

We assessed first the effects of local intraplantar injection of cytoskeletal disruptors on basal nociceptive threshold and on the hyperalgesia induced by carrageenan. None of the compounds used affected basal thresholds, ie, those measured in paws injected with saline, assayed over 8 hours or the absolute values at time zero in groups of treated animals (data not shown). However, carrageenan-induced hyperalgesia was

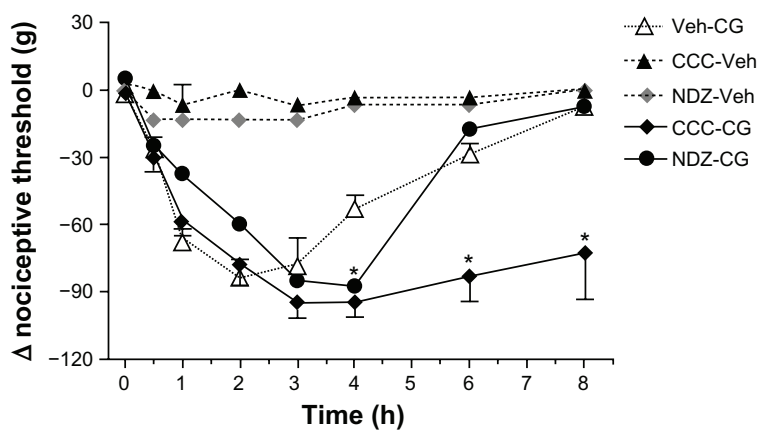

Figure I Colchicine and nocodazole potentiate carrageenan-induced hyperalgesia in rat paws.

Notes: Although neither intraplantar colchicine $8 \mu \mathrm{g}$ administered 60 minutes before intraplantar saline (vehicle) nor intraplantar nocodazole $10 \mu \mathrm{g}$ administered 60 minutes before intraplantar saline affected the nociceptive thresholds in noninflamed paws, they both increased the duration of hyperalgesia induced by intraplantar carrageenan $250 \mu \mathrm{g}$ administered at time zero. Nocodazole extended hyperalgesia by just one hour, but colchicine was more effective, with hyperalgesia prolonged to at least 8 hours after carrageenan injection. Data are shown as the mean \pm standard error of the mean for five rats in each treatment group. $* P<0.05$, significant effect of the cytoskeletal disruptors. The hyperalgesia induced by carrageenan alone was significantly different from basal values for at least 4 hours but has not been marked in the interests of clarity.

Abbreviations: Veh, vehicle; CCC, colchicine; CG, carrageenan; NDZ, nocodazole. 
Table I Effects of cytoskeletal disruptors, given locally, on carrageenan-induced edema in rat paws

\begin{tabular}{|c|c|c|c|c|c|c|}
\hline \multirow[t]{2}{*}{ Treatment } & \multicolumn{6}{|c|}{$\Delta$ paw volume $(\mathrm{mL})$ after carrageenan $(\mathrm{CG})$ injection/time $(\mathrm{h})$} \\
\hline & $\mathbf{0}$ & I & 2 & 3 & 4 & 6 \\
\hline CG only & $0.02 \pm 0.01$ & $0.4 \pm 0.1$ & $0.8 \pm 0.2$ & $0.7 \pm 0.2$ & $0.6 \pm 0.2$ & $0.5 \pm 0.2$ \\
\hline$A C D+C G$ & $0.01 \pm 0.02$ & $0.4 \pm 0.1$ & $0.6 \pm 0.2$ & $0.7 \pm 0.1$ & $0.5 \pm 0.2$ & $0.4 \pm 0.2$ \\
\hline $\mathrm{LAT}+\mathrm{CG}$ & $0.02 \pm 0.02$ & $0.4 \pm 0.1$ & $0.8 \pm 0.3$ & $0.7 \pm 0.2$ & $0.6 \pm 0.2$ & $0.3 \pm 0.1$ \\
\hline $\mathrm{CB}+\mathrm{CG}$ & $0.04 \pm 0.01$ & $0.6 \pm 0.1$ & $0.8 \pm 0.3$ & $0.8 \pm 0.3$ & $0.7 \pm 0.2$ & $0.4 \pm 0.1$ \\
\hline $\mathrm{NCZ}+\mathrm{CG}$ & $0.01 \pm 0.01$ & $0.4 \pm 0.1$ & $0.7 \pm 0.2$ & $0.9 \pm 0 . I^{*}$ & $0.5 \pm 0.1$ & $0.4 \pm 0.1$ \\
\hline $\mathrm{CCC}+\mathrm{CG}$ & $0.04 \pm 0.0 \mathrm{I}$ & $0.6 \pm 0.2$ & $0.9 \pm 0.2^{*}$ & $1.0 \pm 0.2 *$ & $I . I \pm 0.1 *$ & $I . I \pm 0 . I *$ \\
\hline
\end{tabular}

Notes: Edema was assessed by measuring paw volume $(\mathrm{mL})$ for six hours after carrageenan $(\mathrm{CG})$ with a hydroplethysmometer $(\mathrm{Ugo}$ Basile, Italy) and data $(\mathrm{means} \pm \mathrm{SEM})$ are presented as the difference $(\Delta)$ between right and left paw volumes. Carrageenan $(250 \mu \mathrm{g})$ was injected into the rat paw at time zero and the cytoskeleton disruptors acrylamide (ACD; $10 \mu \mathrm{g})$, latrunculin B (LAT; $0.5 \mu \mathrm{g})$, cytochalasin B (CB; I $\mu \mathrm{g})$, nocodazole (NCZ; $10 \mu \mathrm{g})$ or colchicine (CCC; $2 \mu \mathrm{g})$ were given 30 min before the carrageenan (total volume of $50 \mu \mathrm{L}$ per paw). ${ }^{* P}<0.05$, different from corresponding value with $\mathrm{CG}$ only; $\mathrm{N}=4-5$ animals per group.

modified by pretreatment with cytoskeletal disruptors, but only by those affecting microtubule assembly, ie, nocodazole and colchicine (Figure 1). As the time course for these two compounds shows, the peak intensity of hyperalgesia in the early stages (up to 3 hours after carrageenan) was not changed but the duration of hyperalgesia was extended, most clearly by colchicine. The corresponding time courses for the other compounds showed no changes from the time course of carrageenan given alone (data not shown). The contralateral paws, injected with saline instead of carrageenan, did not show changes in nociceptive threshold after carrageenan or after any of the cytoskeletal disruptors (data not shown). The cytoskeletal disruptors produced a comparable profile of effects on the edema induced by carrageenan (Table 1). Only nocodazole or colchicine affected this response and both compounds potentiated or prolonged the increased volume of the inflamed paw. No changes were induced in the volumes of the noninflamed paw by any of the cytoskeletal disruptors tested (data not shown).

\section{Effect of cytoskeletal disruptors on analgesic effects of celecoxib}

We next tested the effects of the cytoskeletal disruptors on the characteristic hypoalgesia induced by celecoxib. The two disruptors of microtubule assembly, nocodazole and colchicine, dose-dependently prevented the hypoalgesic response following systemic administration of celecoxib to rats injected with carrageenan (Figure 2A and B). Both compounds at the higher doses abolished all the analgesic effects of celecoxib, and the nociceptive thresholds after celecoxib and colchicine were almost identical to those seen in the absence of celecoxib (see Figure 1). The disruptors of actin filaments, ie, latrunculin B and cytochalasin B, also achieved dose-dependent reversal of the analgesic effects of celecoxib
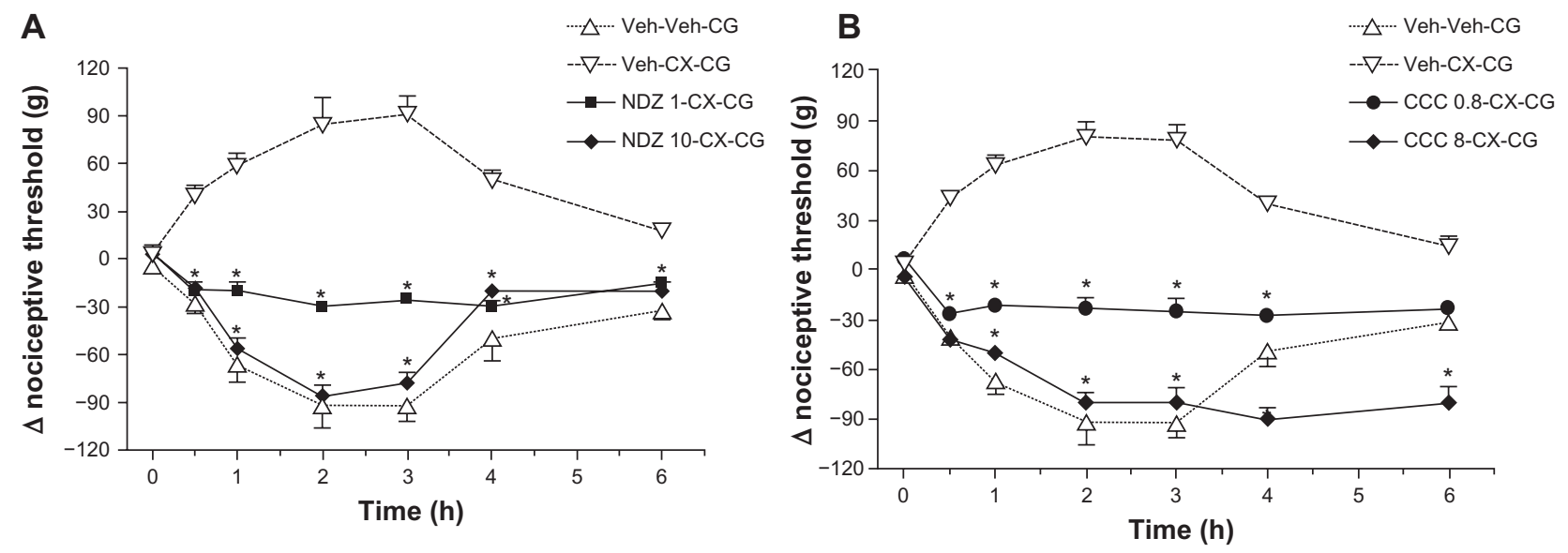

Figure 2 Reversal of celecoxib-induced hypoalgesia by nocodazole or colchicine. Celecoxib $12 \mathrm{mg} / \mathrm{kg}$ administered systemically 30 minutes before carrageenan prevented development of hyperalgesia after carrageenan and induced hypoalgesia ( $\Delta$ nociceptive threshold values greater than zero). In (A), pretreatment with intraplantar nocodazole I $\mu \mathrm{g}$ or $10 \mu \mathrm{g}$ administered 60 minutes before carrageenan dose-dependently reversed the analgesic effects of celecoxib. Data are shown as the mean \pm standard error of the mean for five rats in each treatment group. $* P<0.05$, significant effect of nocodazole. Similarly in (B), intraplantar colchicine $0.8 \mu g$ or $8 \mu g$ administered 60 minutes before carrageenan reversed the effects of celecoxib. Data are shown as the mean \pm standard error of the mean for five rats in each treatment group. $* P<0.05$, significant effects of colchicine.

Abbreviations: CCC, colchicine; CX, celecoxib; NDZ, nocodazole; Veh, vehicle; CE, carrageenan. 
(Figure $3 \mathrm{~A}$ and $\mathrm{B}$ ). It is worth noting that these two compounds were, in molar terms, almost 10 -fold more potent than nocodazole and colchicine in reversing celecoxib-induced antinociception. Pretreatment with acrylamide, a disruptor of intermediary filaments, over a range of doses did not affect the hypoalgesic effect of celecoxib (Figure 3C). Because our experiments showed that cytochalasin B was a potent inhibitor of the hypoalgesia induced by celecoxib and by itself
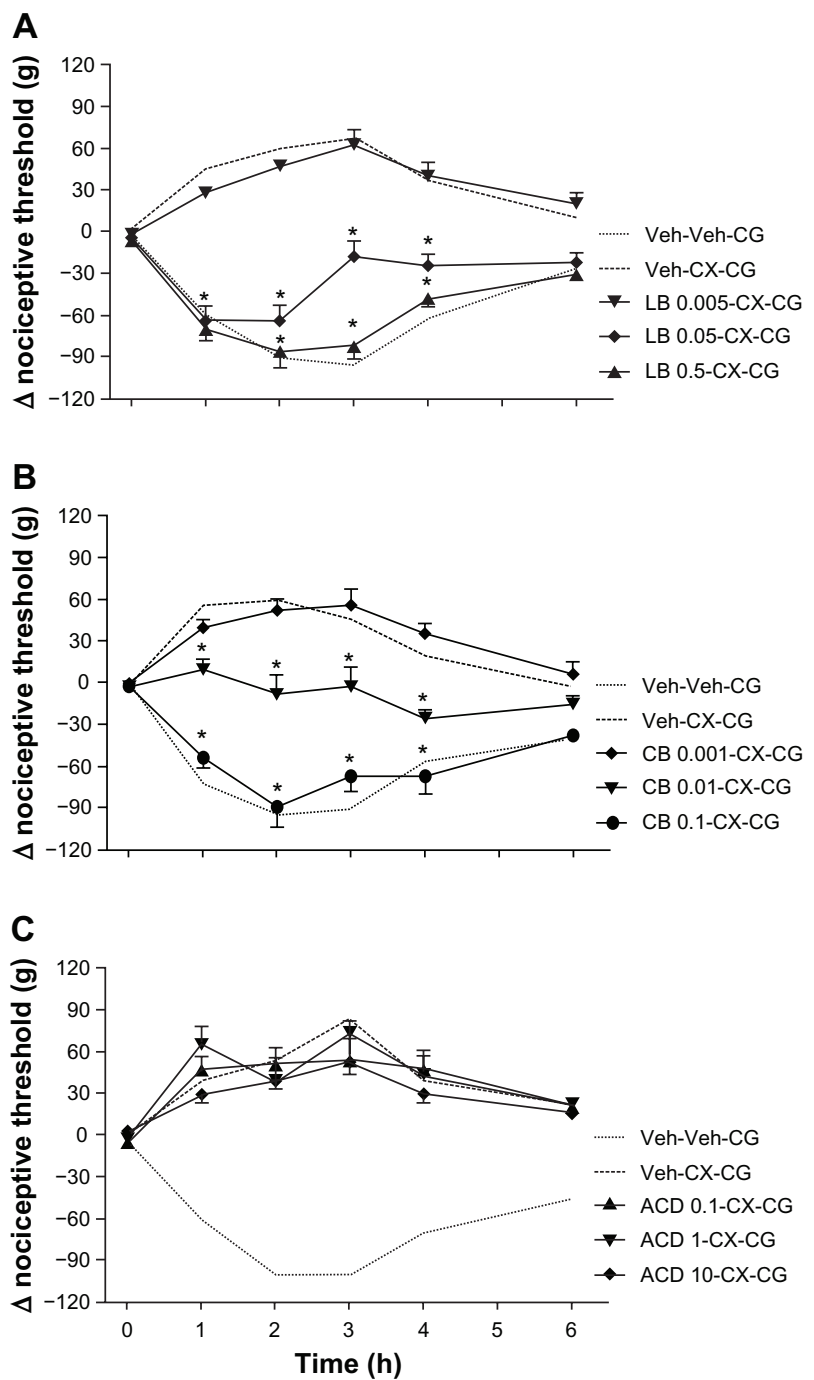

Figure 3 Effects of latrunculin B, cytochalasin B, and acrylamide on celecoxibinduced hypoalgesia. Although intraplantar latrunculin B $0.005-0.5 \mu \mathrm{g}$ administered 60 minutes before carrageenan did not modify the hyperalgesia induced by carrageenan (data not shown), in (A), it dose-dependently reversed the hypoalgesic effects of celecoxib $12 \mathrm{mg} / \mathrm{kg}$ administered systemically 30 minutes before carrageenan. Similarly in (B), intraplantar cytochalasin B $0.00 \mathrm{I}-\mathrm{I} \mu \mathrm{g}$ administered 60 minutes before carrageenan, which did not affect carrageenan-induced hyperalgesia (data not shown), dose-dependently reversed celecoxib-induced hypoalgesia. However, intraplantar acrylamide $0.1-10 \mu \mathrm{g}$ administered 60 minutes before carrageenan neither affected carrageenan-induced hyperalgesia (data not shown) nor, as shown in (C), the corresponding celecoxib-induced hypoalgesia.

Notes: Data are shown as the mean \pm standard error of the mean for five rats in each treatment group. $* P<0.05$, significant effect of cytoskeletal disruptors.

Abbreviations: $A C D$, acrylamide; $C B$, cytochalasin B;CCC,colchicine; $C G$, carrageenan; CX, celecoxib; LB, latrunculin B; Veh, vehicle. did not modify the hyperalgesia induced by carrageenan in rat paws, we used cytochalasin B as a standard cytoskeleton disruptor in our subsequent studies.

\section{Time dependence of celecoxib-induced reversal of hypoalgesia by cytochalasin $B$}

In our first set of experiments with cytochalasin B, we used it as a pretreatment, ie, before either celecoxib or carrageenan. We now gave it after carrageenan, at times when the celecoxib-induced hypoalgesia was developing, at 30 or 60 minutes following carrageenan. As shown in Figure 4, treatment at these later times was less effective in reversing hypoalgesia due to celecoxib. When cytochalasin $\mathrm{B}$ was given at 30 minutes after carrageenan, directly after the assessment of nociceptive threshold, hypoalgesia was already present and 30 minutes later (at one hour after carrageenan) the thresholds were close to those after carrageenan alone, and at all other times there was full reversal of celecoxibinduced hypoalgesia. Given at 60 minutes, at the peak of hypoalgesia, the same dose of cytochalasin B only partly reversed celecoxib-induced analgesia. In addition, at neither of these times was cytochalasin B, given to the contralateral paw, able to modify the nociceptive response to carrageenan or the hypoalgesia induced by celecoxib (data not shown). Phalloidin, a stabilizer of actin filaments, has been shown to block the effects of cytochalasin D in epinephrineinduced paw hyperalgesia. ${ }^{16}$ In our model, similar doses of phalloidin did not affect basal nociceptive thresholds for carrageenan-induced hyperalgesia (data not shown) or

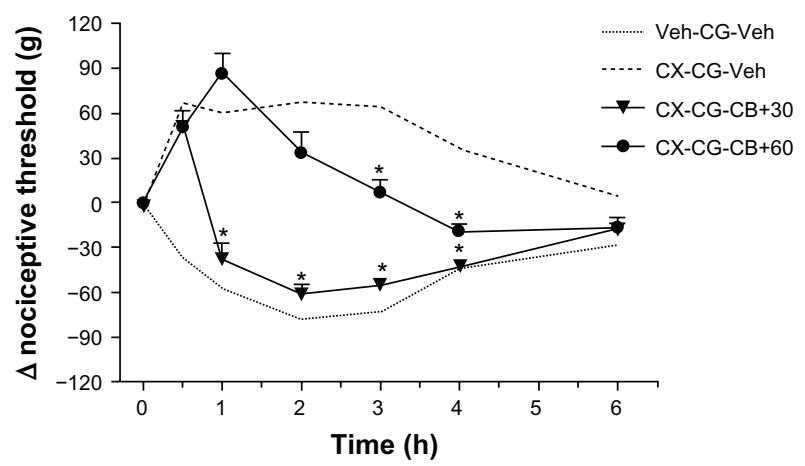

Figure 4 Reversal by cytochalasin B of celecoxib-induced hypoalgesia is dependent on time of treatment.

Notes: In these experiments, intraplantar cytochalasin B I $\mu \mathrm{g}$ was administered 30 or 60 minutes after carrageenan, which corresponded to 60 or 90 minutes after celecoxib $12 \mathrm{mg} / \mathrm{kg}$ systemically. Given at 30 minutes after carrageenan, cytochalasin $B$ was able to reverse the analgesic effects of celecoxib completely from one hour onwards. Later treatment at 60 minutes was less effective, although the effects of celecoxib were still significantly reduced at 2, 3, and 4 hours after carrageenan. The data are shown as the mean \pm standard error of the mean for five rats in each treatment group. $* P<0.05$, significant effect of cytochalasin $B$.

Abbreviations: CG, carrageenan; CX, celecoxib; LB, latrunculin B; Veh, vehicle; $\mathrm{CB}$, cytochalasin $\mathrm{B}$. 
celecoxib-induced hypoalgesia (Figure 5A), but it was able to prevent cytochalasin $\mathrm{B}$ from reversing celecoxib-induced analgesia (Figure 5B). In these experiments, intraplantar cytochalasin B was again given 30 minutes before carrageenan, so that the number of pretreatment injections into the paw was not increased.

\section{Effect of cytochalasin B on antinociceptive response induced by other nonselective and selective COX inhibitors}

To assess the relevance of COX inhibition to the effects of cytochalasin B on celecoxib-induced hypoalgesia, we tested its effects on the analgesia known to be exerted in our model by a variety of COX inhibitors. As shown in Figure 6, pretreatment with a local intraplantar injection of cytochalasin B did not affect the antinociceptive effects exerted by systemic treatment with doses of indomethacin $(4 \mathrm{mg} / \mathrm{kg})$, or the selective inhibitor of COX-1, SC-560 (5 mg/kg). However, hypoalgesia induced by SC-236, another selective inhibitor of COX-2, which is as effective as celecoxib in our model, ${ }^{3-5}$ was reversed by cytochalasin $\mathrm{B}$.

\section{Interactions between cytoskeletal disruptors and opioid system in carrageenan-induced hyperalgesia}

Morphine given as a pretreatment in our model of carrageenaninduced hyperalgesia has a bilateral hypoalgesic effect in rats following intracerebroventricular administration. ${ }^{5,24}$ To assess the effects of cytoskeletal disruption on this hypoalgesia when induced by morphine, we used cytochalasin B, colchicine, and acrylamide. Systemic morphine $2 \mathrm{mg} / \mathrm{kg}$ induced hypoalgesia in both carrageenan-injected (right, inflamed) rat paws (Figure 7A) and saline-injected (left, noninflamed) rat paws (Figure 7B). Colchicine dose-dependently inhibited morphine-induced hypoalgesia and, at the higher dose and at the later times (4 hours onward), produced the now characteristic prolongation of hyperalgesia, up to 8 hours after carrageenan (Figure 7A). Cytochalasin B, over the same dose range that totally reversed celecoxib-induced hypoalgesia was less effective against morphine-induced hypoalgesia, although there was still a very marked decrease in hypoalgesia without complete reversal of all analgesic effects (Figure 7C). Note that neither of these cytoskeletal disruptors, given only to the inflamed paw, affected the response to morphine in the contralateral paw (Figure 7B and D). The disruptor of intermediate filaments, acrylamide, given at $1 \mu \mathrm{g}$ or $10 \mu \mathrm{g}$ to the inflamed paw, did not affect morphine-induced hypoalgesia in either the right or left paws (data not shown).

\section{Discussion}

Our experiments have shown that disruption of the cytoskeleton reversed the analgesia induced by two selective inhibitors of COX-2, ie, celecoxib and SC-236. Such reversal was selective in that not all the cytoskeletal disruptors were equally effective and that the analgesic effects of the nonselective COX inhibitor, indomethacin, and of SC-560, were not affected by any of the cytoskeletal disruptors. However, cytoskeletal disruption also reversed morphine-induced analgesia, strengthening the causal relationship between cytoskeletal structure and the processes involved in nociception and endogenous antinociceptive systems.
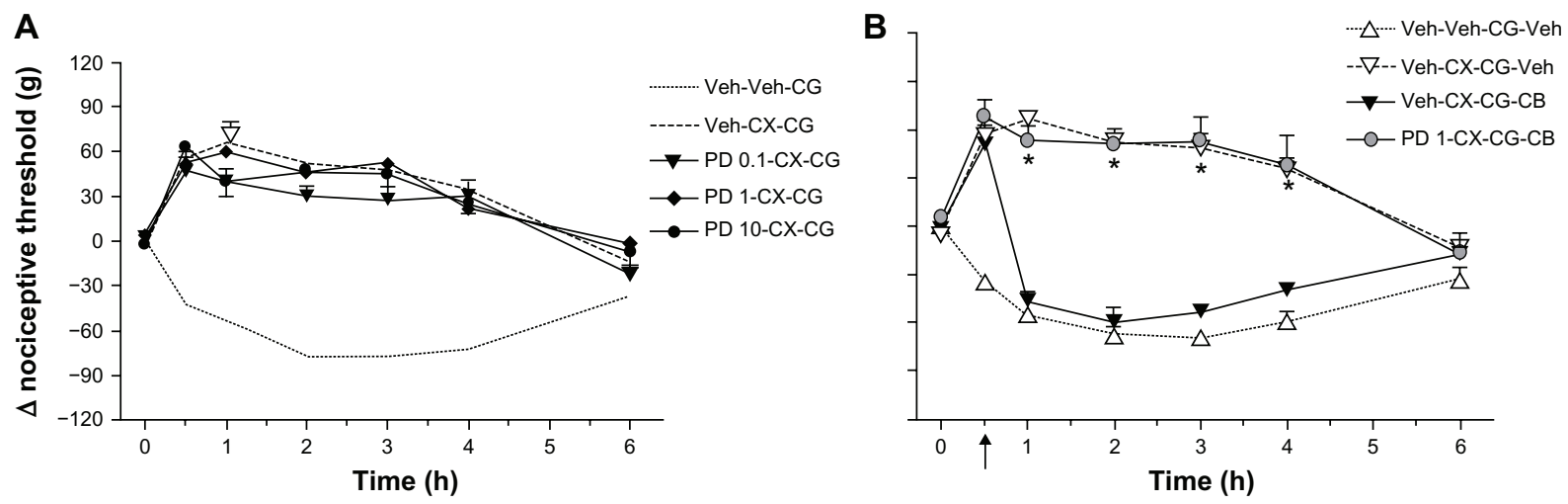

Figure 5 Effects of phalloidin on celecoxib-induced hypoalgesia and interactions with cytochalasin B. In (A), a range of intraplantar doses of phalloidin ( 0 . I- $10 \mu g)$ administered 60 minutes before carrageenan did not modify the hypoalgesia induced by celecoxib $12 \mathrm{mg} / \mathrm{kg}$ administered systemically 30 minutes before carrageenan. The data are shown as the mean \pm standard error of the mean for five rats in each treatment group. In (B), intraplantar cytochalasin B I $\mu g$ administered 30 minutes after carrageenan (see arrow on time axis) reversed all analgesic effects of celecoxib from I-4 hours. Pretreatment with intraplantar phalloidin I $\mu \mathrm{g}$ administered 60 minutes before carrageenan blocked this reversal by cytochalasin $B$. The data are shown as the mean \pm standard error of the mean for five rats in each treatment group.

Note: $* P<0.05$, significant effect of phalloidin.

Abbreviations: CB, cytochalasin B; CG, carrageenan; CX, celecoxib; PD, phalloidin; Veh, vehicle. 


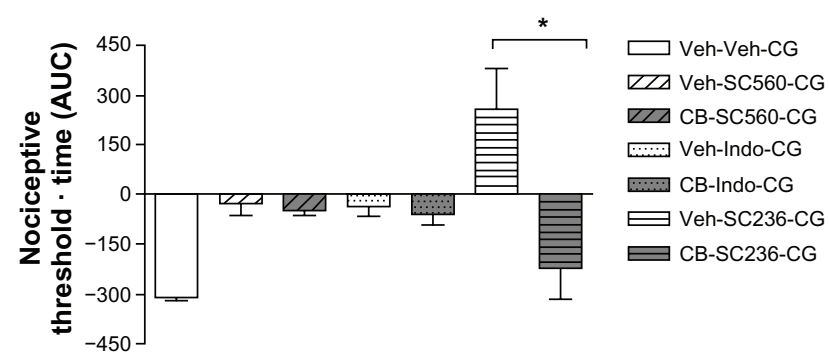

Figure 6 Effects of pretreatment with cytochalasin B on the analgesic effects of other COX inhibitors in carrageenan-induced hyperalgesia.

Notes: The results are summarized here as the AUC of the time course over 6 hours with hyperalgesia represented by negative values of AUC and hypoalgesia by positive values. Carrageenan-induced hyperalgesia (first negative bar) was reversed by the nonselective COX inhibitor, indomethacin, administered at $4 \mathrm{mg} / \mathrm{kg}$ systemically 30 minutes before carrageenan or by the selective inhibitor of COX-1, SC-560 administered at $5 \mathrm{mg} / \mathrm{kg}$ systemically 30 minutes before carrageenan, but only back to basal nociceptive thresholds (AUC about zero). Pretreatment with intraplantar cytochalasin B I $\mu g 60$ minutes before carrageenan did not affect these analgesic effects. However, the selective COX-2 inhibitor, SC-236 $12 \mathrm{mg} / \mathrm{kg}$ administered systemically 30 minutes before carrageenan induced clear hypoalgesia (positive AUC) which was completely reversed by the same pretreatment with cytochalasin B. Data are shown as the mean \pm standard error of the mean for five rats in each treatment group. $* P<0.05$, significant effect of cytochalasin $B$.

Abbreviations: $A \cup C$, area under the concentration-time curve; $C B$, cytochalasin $B$; CG, carrageenan; COX, cyclo-oxygenase; Indo, indomethacin; Veh, vehicle.

Our first experiments evaluating the effects of different cytoskeletal disruptors on the nociceptive thresholds to mechanical stimuli under basal conditions and after inflammation with carrageenan highlighted clear differences between their effects in inflamed paws, although none of them modulated nociceptive thresholds in saline-injected control rat paws. Such control responses were observed either in the left (saline-injected) paw of rats receiving carrageenan in the right paw or in rats receiving saline in only one hind paw. From these results, we deduced that the inflammation was localized, ie, restricted to the injected paw without signs of a systemic response to carrageenan. This lack of effect in control paws would also imply that the cytoskeletal disruptors and, hence, the need for specific cytoskeletal rearrangement, were not critical for the normal nociceptive response to mechanical stimulation in normal noninflamed paws. Our results would agree with those of Dina et al, ${ }^{16}$ who reported a similar lack of modulation of basal nociception by these cytoskeletal disruptors. However, in paws treated with carrageenan, disruptors of microtubules (nocodazole and colchicine) were very clear potentiators of carrageenan-induced hyperalgesia, whereas the others (cytochalasin B, latrunculin B, and acrylamide) did not modify the hyperalgesic effects of carrageenan. Because we measured the time course of hyperalgesia over 6 hours, rather than making a single fixed time assay, we were able to show that the potentiation of carrageenan hyperalgesia was not expressed as a change in intensity of the hyperalgesia, but as an extension of its duration. Our model is capable of showing an increased intensity of hyperalgesia, for instance, to higher doses of carrageenan than those used here. ${ }^{3}$ This increased duration of hyperalgesia might suggest that the major effect of the disrupted microtubules was to influence the resolution of, or recovery from, the hyperalgesic state, rather than its initiation or generation of mediators of hyperalgesia. Such modification of carrageenan-induced inflammation is supported by the effects of the cytoskeletal disruptors on paw edema, where only nocodazole and in particular colchicine prolonged the edema (increased paw volume) in the carrageenan-injected paw. Given that pain and edema are two of the cardinal signs of inflammation, it would appear that disruption of microtubules selectively inhibited resolution of the inflammatory process. This conclusion is in marked contrast with the long established clinical use of systemic colchicine as a potent anti-inflammatory agent. ${ }^{11,25,26}$ This difference is most likely to be related to the different modes of administration; we gave colchicine locally to the paw, whereas in clinical use, colchicine is given orally. Systemic colchicine would clearly have as its first targets circulating leukocytes and endothelial cells, two well recognized sites of the anti-inflammatory actions of colchicine. ${ }^{11}$ Local intraplantar colchicine would access different types of cells and, in particular, relatively few leukocytes and endothelial cells. Interestingly, another potential proinflammatory action of colchicine is its inhibition of steroid biosynthesis, ${ }^{27}$ but such action is probably not relevant over the short experimental period (6 hours) of our model. However, it would be of interest to assess the effects of systemic colchicine on nociception and edema in our model.

Our results with nocodazole and colchicine also contrast with those of Dina et al, ${ }^{16}$ who found these two disruptors to be antinociceptive agents, reducing the hyperalgesia induced by epinephrine. However, these differences may be the result of the different algesic stimuli used (epinephrine, prostaglandin $\mathrm{E}_{2}$, estrogen), either alone or concomitantly, and the extent of the accompanying modulation of inflammation, all of which may involve different intracellular signaling pathways. For instance, peripheral nociception was differentially modulated when the paw was injected with carrageenan or prostaglandin $\mathrm{E}_{2} \cdot{ }^{28}$

We then used a range of known analgesic agents in our model and all clearly decreased the hyperalgesia, with some analgesic agents (celecoxib, SC-236, morphine) inducing a hypoalgesic effect. However, the cytoskeletal disruptors were not all equally effective in modulating these antinociceptive effects. Thus, the disruptors of microtubules or actin were more effective than acrylamide, which did not modify 

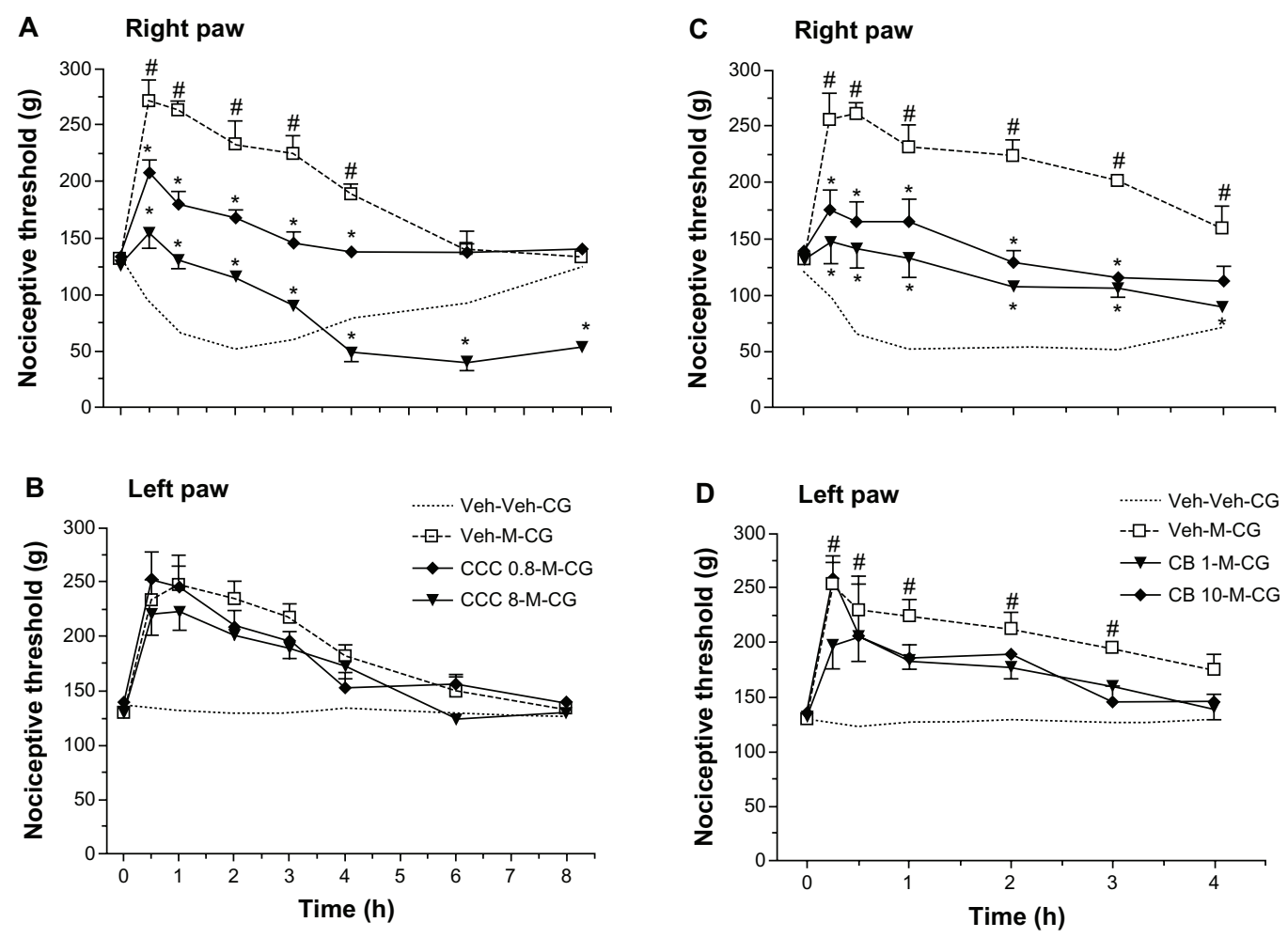

Figure 7 Modulation of morphine-induced hypoalgesia after carrageenan by colchicine or cytochalasin B. In (A), morphine (M) 2 mg/kg administered systemically 30 minutes before carrageenan (CG) was clearly analgesic, changing the hyperalgesia after carrageenan to hypoalgesia. Pretreatment with two doses of intraplantar colchicine (CCC) $0.8 \mu \mathrm{g}$ or $8 \mu \mathrm{g}$ administered at 60 minutes before carrageenan decreased the hypoalgesia but retained the antihyperalgesic effects of morphine. In (B), data from the left paw (without carrageenan) show that hypoalgesia after morphine was not changed by colchicine. Data are shown as the mean \pm standard error of the mean for five rats in each treatment group. Similarly, in (C and D), intraplantar cytochalasin B (CB) I $\mu$ g or $10 \mu g$ administered 60 minutes before carrageenan partly reversed morphine-induced analgesia to antihyperalgesia in the inflamed right paw, without modifying the effects of morphine in the left noninflamed paw. Data are shown as the mean \pm standard error of the mean for five rats in each treatment group.

Notes: $\# P<0.05$, significant hypoalgesia; $* P<0.05$, significant effect of colchicine.

Abbreviations: CB, cytochalasin B; CCC, colchicine; CG, carrageenan; Veh, vehicle.

the antinociceptive effects of any of the analgesic agents used here.

Disruption of actin filaments by cytochalasin B or latrunculin B, although ineffective against carrageenaninduced hyperalgesia, proved to be highly effective in reversing the analgesic effects of celecoxib and SC-236. Moreover, phalloidin, an actin filament stabilizer, ${ }^{12}$ prevented this effect of cytochalasin B, although phalloidin by itself did not affect celecoxib-induced hypoalgesia or the nociceptive response to carrageenan, implying that changes in actin filaments were induced particularly during the hypoalgesic response to celecoxib. A similar "antagonism" between phalloidin and cytochalasin D, another actin disruptor, has been reported in epinephrine-induced hyperalgesia. ${ }^{16}$ The reversal of celecoxib-induced hypoalgesia by the two microtubule disruptors (nocodazole and colchicine) is less easily interpreted, because these compounds potentiated carrageenan-induced inflammation, ie, they prolonged the hyperalgesia and edema in the absence of any analgesic agent. Therefore, our results for nocodazole and colchicine were not immediately helpful in elucidating the mechanism(s) underlying the actions of celecoxib in our model. Nevertheless, low doses of cytochala$\sin \mathrm{B}$ prevented celecoxib-induced hypoalgesia, an action that was completely blocked by phalloidin. This finding, taken together with the lack of effect for the intermediate filament disruptor, acrylamide, on celecoxib-induced hypoalgesia would suggest that actin filaments are crucially involved in the expression of celecoxib-induced hypoalgesia.

Another important outcome of these experiments was the clear division between the two coxibs (celecoxib and SC-236) and the other NSAIDs (indomethacin and SC-560), in terms of their response to cytochalasin B. Because indomethacin and SC-560 are inhibitors of COX, ${ }^{19,29}$ their analgesic activities have been attributed to their ability to block prostaglandin biosynthesis. ${ }^{1}$ Thus, the resistance of analgesic effects to modification by cytochalasin B observed here would be compatible with the resistance of prostaglandin $\mathrm{E}_{2}$-induced paw hyperalgesia to cytochalasin $\mathrm{D}$ and other actin filament disruptors. ${ }^{16}$ If, as proposed by Dina et al, ${ }^{16}$ signaling pathways via protein kinase $\mathrm{A}$ are involved in prostaglandin $\mathrm{E}_{2}$-induced 
hyperalgesia and these are not affected by disruption of actin filaments, then equal resistance would be expected for those analgesics with an action based on lowering prostaglandin production. This division between the coxibs and the other NSAIDs would support the possibility of a nonprostaglandinbased mechanism underlying the celecoxib-induced hypoalgesia in this model, as we have consistently found..$^{3-5,30}$

The profile of effects of the cytoskeletal disruptors on morphine-induced analgesia in our model matched that obtained with celecoxib, insofar as disruptors of actin and microtubules reversed the effects of morphine, whereas acrylamide was ineffective. Although there is evidence of changes in cytoskeletal proteins after chronic morphine treatment in the context of correlating protein modifications with addiction and/or withdrawal, ${ }^{31-33}$ links between acute agonist exposure and proteins have focused on those proteins more closely associated with receptor function, such as $\beta$-arrestin, $G$ protein-coupled inwardly-rectifying potassium (GIRK) channels, and regulators of G-protein signaling. ${ }^{34,35}$ Thus, our results for the acute modulation of morphine-induced analgesia by cytochalasin B may be the first such data from an in vivo model. Although the interactions of cytochalasin B with $\beta$-arrestin, GIRK, and G-protein signaling proteins have not been specifically studied, it is very likely that these cytoskeletal disruptors modulated the acute response to opioid receptor agonists by affecting actin and/or microtubular structures involved in the intracellular processing of such receptors through endosomes, because internalization and recycling of opioid receptors is crucial to their function ${ }^{36}$ and actin polymerization plays a critical role in endocytic function. ${ }^{37,38}$ Recently, rapid recycling of $\mu$-opioid receptors in HEK293 cells and in neurons has been described, which involves a crucial interaction with actin and microtubules. ${ }^{39}$ Disruption of these cytoskeletal components (with latrunculin and nocodazole) decreased recycling of internalized $\mu$-receptors to the cell membrane, and could thus lead to a loss of response to opioid agonists. In our model, such events would lead to a loss of analgesia induced by morphine and endogenous opioids.

An important feature of the action of morphine in this model was that it affected nociceptive thresholds in the contralateral noninflamed paw as well as those in the inflamed paw, as found earlier. ${ }^{5}$ It is worth noting that although morphine and celecoxib were both given systemically, celecoxib only affected thresholds in the inflamed paw, ${ }^{3-5,40}$ and this difference does imply differences in the sites of action between the two compounds. In the present experiments, colchicine and cytochalasin B were relatively less effective against morphine than they were against celecoxib, with some antinociceptive effects persisting for the first few hours after morphine. Bearing in mind that these two disruptors were given locally into the paw, this difference could derive from a difference in action on peripheral versus central sites. Although both morphine and celecoxib are active peripherally and centrally, ${ }^{5,24,41,42}$ morphine is more likely to access the central nervous system rapidly and is a direct agonist at opioid receptors, whereas celecoxib does not readily cross the bloodbrain barrier ${ }^{5,43,44}$ and must act indirectly because it is not an agonist at opioid receptors. ${ }^{6}$ These differences may result in a greater proportion of centrally mediated analgesia for morphine than for celecoxib, when either is given systemically. Only the local peripheral actions of the analgesics would be affected by cytochalasin B given via the intraplantar route, because our assay with cytochalasin B given to the noninflamed paw showed that not enough cytochalasin B circulated to the inflamed paw to modulate nociceptive responses. The less efficient reversal of the effect of morphine probably reflects the greater proportion of its analgesia being mediated via the central nervous system.

One clear limitation of our study is that although we considered the actions of the cytoskeletal disruptors to be restricted to the paw and thus acting peripherally, we do not know which cells in the paw are primarily affected. Given that cytochalasin B reversed morphine-induced analgesia, one site of action for the cytoskeletal disruptors could be cells bearing opioid receptors, most obviously, sensory neurones. Actions on such cells would also explain the reversal by cytochalasin B of celecoxib-induced hypoalgesia, because this hypoalgesia was also blocked by opioid receptor antagonists. ${ }^{4,5,30,40}$ Furthermore, there is cross-tolerance between celecoxib and morphine. ${ }^{45}$ Overall, there is good evidence in our model for celecoxib-induced hypoalgesia being mediated by activation of opioid receptors. However, because celecoxib is not an agonist at opioid receptors, we have postulated an indirect activation of opioid receptors by the release of endogenous opioids. ${ }^{5,30}$ A relevant example of such release is the action of chemokines to release opioids from primary granules in polymorphonuclear leukocytes. ${ }^{46}$ If, in our experimental system, celecoxib does release endogenous opioids from peripheral stores in leukocytes ${ }^{46,47}$ or keratinocytes, ${ }^{48,49}$ then the release process itself would provide another possible locus of action for the cytoskeletal disruptors. The involvement of actin in the release of two other peptides (oxytocin and vasopressin) from hypothalamic neurones ${ }^{50}$ and that of dopamine from PC12 cells ${ }^{51}$ has already been demonstrated. 
There is very recent evidence for a further complication in the mechanism underlying celecoxib-induced hypoalgesia, because celecoxib given intracerebroventricularly also induced hypoalgesia in paws injected with carrageenan, and this central action of celecoxib was blocked by opioid receptor antagonists and by an antagonist of cannabinoid $\mathrm{CB}_{1}$ receptors. ${ }^{30}$ These results would suggest that celecoxib may release endocannabinoids, which in turn, release endogenous opioids, as shown by Ibrahim et al. ${ }^{49}$ If such a link can be demonstrated in the periphery, ie, after systemic administration of celecoxib, then there would be two levels of endogenous transmitter release and action at which cytochalasin B might act. Nevertheless, for either direct or indirect activation of opioid receptors, blockade of the intracellular processes following opioid receptor activation, by interfering with the cytoskeleton, would be sufficient to prevent analgesic effects.

Another limitation is that we have not shown directly that, in our model, the different cytoskeletal components were disrupted by the agents used. Also, several of the disruptors have other cellular effects, apart from any selective effects on the cytoskeleton. For instance, colchicine induces neuronal apoptosis and is antiproliferative, ${ }^{11,52}$ and nocodazole, colchicine, and cytochalasin B block glucose transport. ${ }^{53}$ However, for colchicine and nocodazole, our finding that hyperalgesia and edema were prolonged rather than intensified or blocked suggests a more subtle effect than neuronal toxicity. For cytochalasin B, reversal by phalloidin would at least be compatible with effects on particular components of the cytoskeleton. Similarly, a general metabolic toxicity that could follow blockade of glucose uptake is also an unlikely explanation for our observations, given that glucose transporters are inhibited effectively by either nocodazole, colchicine or cytochalasin $\mathrm{B},{ }^{53}$ whereas in our experiments, these cytoskeletal disruptors exerted clearly different effects. In future work, a direct assessment of the state of the microtubular system and actin in our experimental system should be undertaken.

In summary, our results imply that some, but not all, components of the cytoskeleton are involved in the mechanisms underlying the hyperalgesia and edema characteristic of a well established model of peripheral, localized inflammation. Furthermore, the analgesic effects of morphine and celecoxib, but not those of indomethacin, were also affected by disruption of the cytoskeleton. Overall, our results provide further evidence for the critical and causal involvement of the cytoskeleton in expression of inflammatory pain and of endogenous antinociceptive systems triggered by such stimuli. They also support our proposal that the mechanisms underlying the antinociceptive actions of celecoxib in this model involve the endogenous opioid system.

\section{Acknowledgments}

This work was supported by the Conselho Nacional de Pesquisa (National Council for Scientific and Technological Development [CNPq]), Coordenação de Aperfeiçoamento de Pessoal de Nível Superior (Coordination for the Improvement of Higher Level Personnel [CAPES]), and the Fundação de Amparo à Pesquisa de Minas Gerais (The State of Minas Gerais Research Foundation [FAPEMIG]). The authors also acknowledge the excellent technical assistance of Webster Glayser Pimenta dos Reis.

\section{Disclosure}

The authors report no conflicts of interest in this work.

\section{References}

1. Vane JR, Bakhle YS, Botting RM. Cyclooxygenases 1 and 2. Annu Rev Pharmacol Toxicol. 1998;38:97-120.

2. Penning TB, Talley JJ, Bertenshaw SR, et al. Synthesis and biological evaluation of the 1,5-diarylpyrazole class of cyclooxygenase-2 inhibitors: identification of 4-[5-(4-methylphenyl)-3(trifluoromethyl)-1H-pyrazol-1-y1] benzenesulfonamide (SC-58635, Celecoxib). J Med Chem. 1997;40:347-365.

3. Francischi JN, Chaves CT, Lima AS, Bakhle YS. A new anti-nociceptive pathway mediated by cyclooxygenase- 2 in rat paw inflammation induced by carrageenan. Br J Pharm. 2002;135:159.

4. França DS, Ferreira-Alves DL, Duarte ID, et al. Endogenous opioids mediate the hypoalgesia induced by inhibitors of cyclooxygenase- 2 in rat paws treated with carrageenan. Neuropharmacology. 2006;51: $37-43$.

5. Rezende RM, Reis WGP, Duarte ID, Lima PP, Bakhle YS, Francischi JN. The analgesic actions of centrally administered celecoxib are mediated by endogenous opioids. Pain. 2009;142:94-100.

6. Gregori-Puigjané E, Mestres J. A ligand-based approach to mining the chemogenomic space of drugs. Comb Chem High Throughput Screen. 2008;11:669-676.

7. Bhave G, Gereau RW. Growing pains: cytoskeleton as a critical regulator of pain plasticity. Neuron. 2003;39:577-583.

8. Hucho T, Levine JD. Signalling pathways in sensitization: towards a nociceptor cell biology. Neuron. 2007;55:365-376.

9. Eisenhut M, Wallace H. Ion channels in inflammation. Pflugers Arch. 2011;461:401-421.

10. Samson F, Donoso JA, Bettinger IH, Watson D, Himes RH. Nocodazole action on tubulin assembly, axonal ultrastructure and fast axoplasmic transport. J Pharmacol Exp Ther. 1979;208:411-417.

11. Terkeltaub RA. Colchicine update: 2008. Semin Arthritis Rheum. 2008;38:411-419.

12. Cooper JA. Effects of cytochalasin and phalloidin on actin. J Cell Biol. 1987;105:1473-1478.

13. Spector I, Shochet NR, Blasberger D, Kashman Y. Latrunculins novel marine macrolides that disrupt microfilament organization and affect cell growth: I. Comparison with cytochalasin D. Cell Motil Cytoskeleton. 1989;13:127-144.

14. Wakatsuki T, Schwab B, Thompson NC, Elson EL. Effects of cytochalasin D and latrunculin B on mechanical properties of cells. J Cell Sci. 2001;114:1025-1036.

15. LoPachin RM, Ross JF, Lehning EJ. Nerve terminals as the primary site of acrylamide action: a hypothesis. Neurotoxicology. 2002;23:43-59. 
16. Dina AO, McCarter GC, Coupade C, Levine JD. Role of the sensory neuron cytoskeleton in second messenger signalling for inflammatory pain. Neuron. 2003;39:613-624.

17. Di Rosa M. Biological properties of carrageenan. J Pharm Pharmacol. 1972;24:89-102.

18. Vinegar R, Truax JF, Selph JL, Johnston PR, Venable AL, McKenzie KK. Pathway to carrageenan-induced inflammation in the hind limb of the rat. Fed Proc. 1987;46:118-126.

19. Smith CJ, Zhang Y, Koboldt CM, et al. Pharmacological analysis of cyclooxygenase-1 in inflammation. Proc Natl Acad Sci U SA. 1998;95: 13313-13318.

20. Francischi JN, Paiva-Lima P, França DS, Bakhle YS. Crucial role of the cytoskeleton in hypoalgesia induced by celecoxib in rat inflammatory pain. In: VIIIth World Congress of Inflammation, Copenhagen. Inflammation Res. 2007;56:389.

21. Zimmermann M. Ethical guidelines for investigations of experimental pain in conscious animals. Pain. 1983;16:109-110.

22. Rezende RM, França DS, Menezes GB, Dos Reis WPG, Bakhle YS, Francischi JN. Different mechanisms underlie the analgesic actions of paracetamol and dipyrone in a rat model of inflammatory pain. $\mathrm{Br} J$ Pharmacol. 2008;53:760-768.

23. Randall LD, Selitto JJ. A method for measurement of analgesic activity on inflamed tissues. Arch Int Pharmacodyn Ther. 1957;113:233-249.

24. Ferreira SH, Lorenzetti BB, Corrêa FMA. Central and peripheral antialgesic action of aspirin-like drugs. Eur J Pharmacol. 1978;53:39-48.

25. Ahern MJ, Reid C, Gordon TP, McCredie M, Brooks PM, Jones M. Does colchicine work? The results of the first controlled study in acute gout. Aust N Z J Med. 1987;17:301-304.

26. Das SK, Ramakrishnan S, Mishra K, et al. A randomized controlled trial to evaluate the slow-acting symptom-modifying effects of colchicine in osteoarthritis of the knee: a preliminary report. Arthritis Rheum. 2002;47:280-284.

27. Sewer MB, Li D. Regulation of steroid hormone biosynthesis by the cytoskeleton. Lipids. 2008;43:1109-1115.

28. Alves DP, Motta PG, Paiva-Lima P, et al. Inflammation mobilizes local resources to control hyperalgesia: the role of endogenous opioid peptides. Pharmacology. 2012;89:22-28.

29. Vane JR. Inhibition of prostaglandin synthesis as a mechanism of action for aspirin-like drugs. Nat New Biol. 1971;231:232-235.

30. Rezende RM, Lima PP, Reis WGP, et al. Endogenous opioid and cannabinoid mechanisms are involved in the analgesic effects of celecoxib in the central nervous system. Pharmacology. 2012;89:127-136.

31. Loguinov AV, Anderson LM, Crosby GJ, Yukhananov RY. Gene expression following acute morphine administration. Physiol Genomics. 2001;6:169-181.

32. Marie-Claire C, Courtin C, Roques BP, Noble F. Cytoskeletal genes regulation by chronic morphine treatment in rat striatum. Neuropsychopharmacology. 2004;29:2208-2215.

33. Christie MJ. Cellular neuroadaptations to chronic opioids: tolerance, withdrawal and addiction. Br J Pharmacol. 2008;154:384-396.

34. González-Rodrigues S, Hidalgo A, Baamonde A, Menéndez L. Involvement of $\mathrm{Gi} / \mathrm{o}$ proteins and GIRK channels in the potentiation of morphine-induced spinal analgesia in acutely inflamed mice. Naunyn Schmiedebergs Arch Pharmacol. 2010;381:59-71.

35. Fan XL, Zhang JS, Zhang XQ, Yue W, Ma L. Differential regulation of beta-arrestin 1 and beta-arrestin 2 gene expression in rat brain by morphine. Neuroscience. 2003;117:383-389.

Journal of Pain Research

\section{Publish your work in this journal}

The Journal of Pain Research is an international, peer-reviewed, open access, online journal that welcomes laboratory and clinical findings in the fields of pain research and the prevention and management of pain. Original research, reviews, symposium reports, hypothesis formation and commentaries are all considered for publication.
36. Nagi K, Piñeyro G. Regulation of opioid receptor signalling: implications for the development of analgesic tolerance. Mol Brain. 2011;4:25.

37. Putthenveedu MA, Lauffer B, Temkin P, et al. Sequence-dependent sorting of recycling proteins by actin-stabilized endosomal microdomains. Cell. 2010;143:761-773.

38. Skruzny M, Brach T, Ciuffa R, Rybina S, Wachsmuth M, Kaksonen M. Molecular basis for coupling the plasma membrane to the actin cytoskeleton during clathrin mediated endocytosis. Proc Natl Acad Sci USA. 2012;109:E2533-E2542.

39. Roman-Vendrell C, Yu YJ, Yudowski GA. Fast modulation of $\mu$-opioid receptor (MOR) recycling is mediated by receptor agonists. J Biol Chem. 2012;287:14782-14791.

40. Correa JD, Paiva-Lima P, Rezende RM, et al. Peripheral mu-, kappa- and delta-opioid receptors mediate the hypoalgesic effect of celecoxib in a rat model of thermal hyperalgesia. Life Sci. 2010;86: 951-956.

41. Sofia RD, Vassar HB, Knobloch LC. Comparative analgesic activity of various naturally occurring cannabinoids in mice and rats. Psychopharmacologia. 1975;40:285-295.

42. Labuz D, Mousa AS, Schäfer M, Stein C, Machelska H. Relative contribution of peripheral versus central opioid receptors to antinociception. Brain Res. 2007;1160:30-38.

43. Bingham S, Beswick PJ, Bountra C, et al. The cyclooxygenase-2 inhibitor GW406381X [2-(4-ethoxyphenyl)-3-[4-(methylsulfonyl) phenyl]-pyrazolo[1,5- $b$ ]pyridazine] is effective in animal models of neuropathic pain and central sensitization. J Pharmacol Exp Ther. 2005;312:1161-1169.

44. Dembo G, Park SB, Kharasch ED. Central nervous system concentrations of cyclooxygenase-2 inhibitors in humans. Anesthesiology. 2005;102:409-415.

45. Rezende RM, Paiva-Lima P, Camêlo VM, Dos Reis WGP, Bakhle YS, Francischi JN. Celecoxib induces tolerance in a model of peripheral inflammatory pain in rats. Neuropharmacology. 2010;59:551-557.

46. Rittner HL, Labuz D, Schaefer M, et al. Pain control by CXCR2 ligands through $\mathrm{Ca}^{2+}$-regulated release of opioid peptides from polymorphonuclear cells. FASEB J. 2006;20:2627-2629.

47. Stein C, Gramsch C, Herz A. Intrinsic mechanisms of antinociception in inflammation: local opioid receptors and $\beta$-endorphin. $J$ Neurosci. 1990;10:1292-1298.

48. Khodorova A, Navarro B, Jouaville LS, et al. Endothelin B receptor triggers an endogenous analgesic cascade at sites of peripheral injury. Nat Med. 2003;9:1055-1061.

49. Ibrahim MM, Porreca F, Lai J, et al. CB2 cannabinoid receptor activation produces antinociception by stimulating peripheral release of endogenous opioids. Proc Natl Acad Sci U S A. 2005;102:3093-3098.

50. Tobin VA, Ludwig M. The role of the actin cytoskeleton in oxytocin and vasopressin release from rat supraoptic nucleus neurons. J Physiol. 2007;582:1337-1348.

51. Lu J, Katano T, Uta D, Furue H, Ito S. Rapid S-nitrosylation of actin by NO-generating donors and in inflammatory pain model mice. Mol Pain. 2011;7:101.

52. Huang $\mathrm{X}$, Cheng Z, Su Q, et al. Neuroprotection by nicotine against colchicine-induced apoptosis is mediated by PI3-kinase-Akt pathways. Int J Neurosci. 2012;122:324-332.

53. Zheng Y, Sarr MG. Translocation of transfected GLUT2 to the apical membrane in rat intestinal IEC-6 cells. Dig Dis Sci. 2012;57: 1203-1212.

\section{Dovepress}

The manuscript management system is completely online and includes a very quick and fair peer-review system, which is all easy to use. Visit http://www.dovepress.com/testimonials.php to read real quotes from published authors. 